\title{
Typical clinical doses of methylphenidate did not cause tics or exacerbate pre-existing tics in children with attention deficit hyperactivity disorder
}

Law SF, Schachar RJ. Do typical clinical doses of methylphenidate cause tics in children treated for attention-deficit hyperactivity disorder? J Am Acad Child Adolesc Psychiatry 1999 Aug;38:944-51.

QUESTION: In children treated for attention deficit hyperactivity disorder (ADHD), do typical clinical doses of methylphenidate (MPH) cause tics or exacerbate pre-existing mild to moderate tics?

\section{Design}

Non-randomised, blinded (patients and outcome assessors)*, placebo controlled trial with 1 year of follow up. Children were originally randomised to the intervention groups but crossovers were maintained and analysed in the group they crossed over to.

\section{Setting}

The Hospital for Sick Children, Toronto, Ontario, Canada.

\section{Patients}

91 children (mean age 8 y, $81 \%$ boys) with ADHD with and without comorbid tics who had no previous medication for ADHD or tics. Children had to have a history of ADHD symptoms for $\geqslant 6$ months beginning before the age of 7 years and an estimated Full Scale Intelligence Quotient $>80$. Exclusion criteria were severe tics or Tourette's syndrome, or primary anxiety or affective disorder.

\section{Assessment of risk factors}

46 children were allocated to MPH (the dose was titrated to balance behaviour change and side effects in $5 \mathrm{mg}$ steps to a target dose of $0.7 \mathrm{mg} / \mathrm{kg}$ body weight twice daily) and 45 were allocated to placebo. 27 children allocated to placebo crossed over to MPH (none due to the onset of tic disorder), and 1 child assigned to MPH dropped out (none crossed over to placebo). The final MPH group had 72 children and the placebo group had 18.

\section{Main outcome measures}

Occurrence or exacerbation of tics assessed by interviewing parents and teachers.

\section{Main results}

The mean dose of MPH was $0.5 \mathrm{mg} / \mathrm{kg}$ twice daily. Clinically significant tics developed in $20 \%$ of children (10 out of 51 children) without pre-existing tics in the MPH group compared with 17\% (2 out of 12) of those receiving placebo (relative risk [RR] 1.2, 95\% CI 0.3 to $4.4, \mathrm{p}=0.6$ ). Deterioration of tics was observed in $33 \%$ of children (7 out of 21) with pre-existing tics in the MPH group compared with $33 \%$ (2 out of 6 ) of those in the placebo group (RR 1.0, CI 0.4 to $1.9, \mathrm{p}=0.7$ ). The study had a power of $81 \%$ to detect a substantive risk associated with MPH use (effect size 0.30 ) at a $0.05 \alpha$ level.

\section{Conclusion}

In children treated for attention deficit hyperactivity disorder, typical clinical doses of methylphenidate did not cause tics or exacerbate pre-existing mild to moderate tics.

*See glossary.

\section{COMMENTARY}

Law and Schachar corroborated findings from previous studies by Gadow et al and Castellanos et al showing that MPH did not lead to an increase in tics in children with ADHD. ${ }^{12}$ The strengths of this study are: patients had no previous medication exposure; the placebo control was continued over a 1 year period; and the prescribing method closely mirrored good clinical practice (ie, dosage titration according to symptom response and side effects, allowing for crossing over in treatment non-responders).

Although the results are encouraging for the safety of MPH use, several questions remain. The study was unable to assess the relation between MPH dose and the emergence of tics. Previous studies have postulated that high doses of MPH might be associated with tic emergence. ${ }^{2}$ Furthermore, an increase in tics during MPH use may have been underestimated due to 2 important factors. Firstly, the small placebo control group reduces the likelihood of finding drug-placebo differences. The power calculations do not appear to take into account unequal groups due to large numbers of patients crossing over to MPH treatment. Secondly, the measurement of tics used teacher and parent reports that were noted to be highly discrepant. Previous studies have used objective measurement of tics such as videotaped simulated classroom evaluations with good inter-rater reliability. ${ }^{12}$ Objective measurement techniques eliminate error associated with potentially biased subjective reports.

As Law and Schachar have indicated, further studies are needed to clarify these questions. This study does, however, add to the evidence on the safety of stimulants. The prescribing methods used can be readily applied to clinical practice.

Chiachen Cheng, MD Mark Sanford, MD, ChB, FRCP(C) McMaster University Hamilton, Ontario, Canada

1 Gadow KD, Sverd J, Sprafkin J, et al. Long-term methylphenidate therapy in children with comorbid attention-deficit hyperactivity disorder and chronic multiple tic disorder. Arch Gen Psychiatry 1999;56:330-6.

2 Castellanos FX, Giedd JN, Elia J, et al. Controlled stimulant treatment of ADHD and comorbid Tourette's syndrome: effects of stimulant and dose. J Am Acad Child Adolesc Psychia try 1997;36:589-96.
Source of funding: Medical Research Council of Canada.

For correspondence: Dr R Schachar, Child Psychiatry Research Unit, The Hospital for Sick Children, Toronto, ON M5G $1 X 8$ Canada.Fax +1416 8136565 . 